\title{
Associação e poder discriminatório da atividade física para a prevenção da sarcopenia em mulheres pós-menopáusicas
}

\section{Association and discriminatory power of physical activity for the prevention of sarcopenia in postmenopausal women}

\author{
Cristiano Penas Seara Pitanga ${ }^{*}$, Francisco José Gondim Pitanga², Ronaldo Eugênio \\ Calçada Dias Gabriel ${ }^{3}$, Maria Helena Rodrigues Moreira ${ }^{3}$
}

ARTIGO ORIGINAL | ORIGINAL ARTICLE

\begin{abstract}
A sarcopenia é frequentemente observada na pós-menopausa e as causas incluem alterações no estado hormonal e níveis reduzidos de atividade física. Prejuízo funcional e incapacidade física são as principais consequências desta condição. O estudo procurou analisar a associação e o poder discriminatório da atividade física (AF) para a prevenção da sarcopenia em mulheres pós-menopáusicas. A amostra inclui 257 mulheres $(57.2 \pm 6.6$ anos; $69.1 \pm 11.3 \mathrm{~kg} ; 155.0 \pm 5,2 \mathrm{~cm})$ e a composição corporal foi medida por bioimpedância. A versão longa do International Physical Activity Questionnaire foi utilizada na apreciação dos níveis de atividade física habitual. A associação das variáveis foi testada através de regressão logística e o poder discriminatório através das curvas Receiver Operating Characteristic, considerando um intervalo de confiança de $95 \%$. A presença de um dispêndio calórico semanal de 580 a $816 \mathrm{kcal}$ através da prática de caminhada revelou poder discriminatório para uma melhor condição muscular na pós-menopausa, sendo registada uma associação significativa entre este tipo de AF e a sarcopenia apenas para o valor de corte de $816 \mathrm{kcal} / \mathrm{semana}$. Os resultados do estudo sugerem que a prática de caminhada, reunindo um dispêndio energético semanal de $816 \mathrm{kcal}$, previne a sarcopenia em mulheres pós-menopáusicas.
\end{abstract}

Palavras-chave: Atividade física, massa muscular, menopausa.

ABSTRACT

The sarcopenia is frequently observed in postmenopausal women and causes include changes in the hormonal status and low levels of physical activity. Functional impairment and disability are the major consequences of this condition. The study aimed to analyze the association and the discriminatory power of physical activity (PA), for the prevention of sarcopenia in postmenopausal women. The sample includes 257 women $(57.2 \pm 6.6$ years; $69.1 \pm 11.3 \mathrm{~kg} ; 155.0 \pm 5.2 \mathrm{~cm})$ and body composition was measured by bioelectrical impedance. The long version of the International Physical Activity Questionnaire was used for assessing the levels of habitual physical activity. The association of variables was been tested using logistic regression and the discriminatory power through Receiver Operating Characteristic, considering a confidence interval of $5 \%$. The presence of a weekly caloric expenditure $580-816 \mathrm{kcal}$ through the practice of walking proved to be associated with a better muscle condition in postmenopausal, being registered a significant association between this type of physical activity and sarcopenia only for the cutoff value of $816 \mathrm{kcal} /$ week. The study results suggest that the practice of walking, gathering a weekly energy expenditure of $816 \mathrm{kcal}$, prevents sarcopenia in postmenopausal women.

Keywords: Physical activity, muscle mass, menopause.

Artigo recebido a 01.08.2014; Aceite a 22.05.2015

${ }^{1}$ Laboratório Baiano de Ciências do Esporte (SPORTCLIN - BA, Brasil).

${ }^{2}$ Universidade Federal da Bahia, Salvador, Brasil

${ }^{3}$ Universidade de Trás os Montes e Alto Douro (UTAD), Vila Real, Portugal

* Autor correspondente: R. Luís Viana Filho - Itapuã, Salvador - BA, 41630-340, Brasil, E-mail: cpspitanga@gmail.com 


\section{INTRODUÇÃO}

A sarcopenia é caraterizada pela presença de um reduzida massa muscular associada ao comprometimento da função do músculo (força ou desempenho físico), espelhando a influência de distintos fatores entre os quais se incluem o envelhecimento (hormonas sexuais, apoptose, disfunção mitocondrial), o desuso (imobilidade, limitados níveis de atividade física habitual), a inadequada nutrição/absorção dos nutrientes, a presença de fatores endócrinos (resistência insulínica, anormal função da tiroide), a frequência de doenças neuro-degenerativas (perda de neurónios motores) e a caquexia (Cruz-Jentoft et al., 2010).

O declínio anual da massa muscular é particularmente evidente após os 50 anos entre $0.6 \%$ (Aloia, McGowan, Vaswani, Ross, \& Cohn, 1991) e 2\% (Rolland \& Vellas, 2009) e particularmente nos primeiros três anos após a instalação da menopausa (Maltais, Desroches, \& Dionne, 2009), sendo as fibras musculares glicolíticas as mais afetadas, com consequências adversas na força e potência muscular (Rolland $\&$ Vellas, 2009). Segundo Clark e Taylor (2011), após os 65 anos e a cada 5 anos a diminuição da força muscular situa-se entre $4.5 \%$ e $5.5 \%$, traduzindo não apenas a restrição das fibras musculares mas também a deficiente capacidade de ativação dos neurónios motores pelo sistema nervoso, especialmente nos maiores grupos musculares proximais.

Derivado da presença de uma maior esperança de vida e de uma inferior massa muscular da mulher, comparativamente ao género masculino, e considerando ainda os limitados níveis de atividade física habitualmente registados nesta população, a sarcopenia representa um potencial problema de saúde pública na mulher pós-menopáusica, originando limitações na sua mobilidade, obstáculos na realização das tarefas do dia-a-dia, incremento do risco de queda (Boirie, 2009), resistência insulínica (Barsalani, Brochu, \& Dionne, 2013) e maior severidade da sintomatologia do climatério (Lee \& Lee, 2013). Quando a sarcopenia coabita com a sobrecarga ponderal patológica, particularmente com a obesidade central, tende a afetar também a aptidão física funcional (Moreira et al., 2008), a condição óssea (Vaidya, 2014), os parâmetros biomecânicos de pressão plantar (Monteiro et al., 2010) e o risco cardiometabólico (Chung, Kang, Lee, Lee, \& Lee, 2013) da mulher pósmenopáusica.

Segundo Vilaça et al. (2014), apesar das mulheres idosas obesas e ativas fisicamente evidenciarem maior quantidade de massa magra em relação às eutróficas esta caraterística não se revela suficiente na preservação da qualidade muscular (força muscular/massa magra) e da aptidão aeróbia e muscular. Assim, a presença de hábitos de exercício na meia-idade é um fator protetor contra a sarcopenia numa idade mais avançada, sendo efetiva na preservação da massa muscular, da força e da performance do músculo. O treino de força melhora a sensibilidade à insulina e reforça a síntese proteica miofibrilar devendo ser combinado com o exercício cardiovascular e associado a um adequado consumo proteico de cerca de 1.2 $\mathrm{g} / \mathrm{kg}$ de peso/dia (Cruz-Jentoft et al., 2010). Pode-se evidenciar (Bann et al., 2014) que maiores níveis de atividade física/dispêndio energético estão articulados a uma melhor massa e força muscular apendicular na mulher, sugerindo-se a necessidade de cumprimento de níveis de intensidade moderada-vigorosa. Segundo Kemmler e von Stengel (2013), uma frequência de exercício de pelo menos duas sessões por semana é determinante na condição muscular da idosa.

Apesar da destacada prevalência da sarcopenia entre os idosos, esta síndrome permanece ainda pouco reconhecida na prática clínica, carecendo de um apropriado diagnóstico e tratamento. $\mathrm{O}$ desenvolvimento de estudos que contribuam para a melhoria da sua prevenção e terapêutica (Nedergaard, Henriksen, Karsdal, \& Christiansen, 2013) e a identificação das componentes da prescrição de exercício (tipo, intensidade, duração) direcionadas para a preservação da condição músculo-esquelética e da sua função na mulher pós-menopáusica revelam-se de grande importância (Bann et al., 2014). Os estudos que avaliam os níveis de atividade física habitual nesta população através do Internacional Physical Activity 
Questionnaire (IPAQ) examinam particularmente a sua relação com a sintomatologia do climatério (Canário et al., 2012; Tan, Kartal, \& Guldal, 2014) e com a qualidade de vida (Gonçalves et al., 2011; Vagetti et al., 2015), sendo mais limitadas as pesquisas que relacionam a atividade física com a composição corporal (Wee et al., 2013). Neste âmbito, o estudo agora apresentado teve como objetivo analisar a associação e o poder discriminatório da atividade física, avaliada através do IPAQ para prevenção da sarcopenia em mulheres nesta etapa do envelhecimento reprodutivo.

\section{MÉTODO}

O estudo integra o projeto "Menopausa em Forma”, aprovado pela Fundação para a Ciência e Tecnologia (POCI/DES/59049/2004) e pela Universidade de Trás-os-Montes e Alto Douro (UTAD, Portugal) e concebido para analisar os efeitos do exercício no risco cardiovascular e de queda e na aptidão física e funcional de mulheres pós-menopáusicas.

As participantes integraram voluntariamente o projeto em resposta a várias ações de divulgação do mesmo (anúncios de jornal, panfletos distribuídos na comunidade, reportagens na televisão, entre outros) ou foram encaminhadas pelo médico de família. O recrutamento da amostra foi realizado entre novembro de 2005 e março de 2006 e a inclusão no estudo foi precedida da avaliação da história clinica e reprodutiva. O estudo atendeu aos procedimentos da Declaração de Helsínquia (World Medical Association, 2013), tendo sido obtido de todas as participantes o consentimento informado assinado. $\mathrm{O}$ protocolo de pesquisa não foi submetido à Comissão de Ética da UTAD pelo facto deste órgão colegial ter sido estabelecido apenas em 2012.

As avaliações foram conduzidas no Laboratório de Aptidão Física, Exercício e Saúde da UTAD por dois técnicos treinados e supervisionados pela investigadora responsável do projeto.

\section{Amostra}

A amostra incluiu 257 mulheres pósmenopáusicas com média de idade de $57.2 \pm$ 6.6 anos. Os critérios de inclusão observados foram os seguintes: (a) ausência de menopausa precoce; (b) inexistência de significativa doença renal, hepática ou hematológica; (c) não existência de sintomas de angina de peito ou de enfarte do miocárdio nos últimos 3 meses; (d) ausência de uma hipertensão descontrolada (pressão arterial sistólica $\geq 200 \mathrm{mmHg}$ e/ou pressão arterial diastólica $\geq 105 \mathrm{mmHg}$ ); (e) não utilização de medicação $\beta$-bloqueadora ou antiarrítmica e; (f) inexistência de condições músculo-esqueléticas passíveis de condicionarem a prática de exercício ou serem exacerbadas pela mesma.

A menopausa foi classificada de natural, quando o termo dos ciclos menstruais e ovulatórios decorreu sem causa patológica evidente, existindo uma amenorreia permanente há pelo menos 12 meses. O tempo de menopausa ( $1, \mathrm{TM} \leq 10$ anos; $2, \mathrm{TM}>10$ anos), a utilização de terapia hormonal ( $O$, não documenta uso de $\mathrm{TH}$; 1 , documenta o uso de $\mathrm{TH}$ ), o uso de métodos contracetivos ( $O$, não utilizou; 1 , utilizou) e a regularidade dos ciclos menstruais no estádio reprodutivo $(0$, não; 1 , sim) foram expressos numa escala ordinal. Esta última variável foi definida pela presença de ciclos menstruais consistentes com uma duração entre 21 e 35 dias

\section{Instrumentos e Procedimentos}

A medição da altura (ALT) foi efetuada com o estadiómetro SECA 220 (Seca Corporation, Hamburg Germany), sendo cumpridos os procedimentos definidos por Heyward e Wagner (2004) e considerado um limite de tolerância de $2 \mathrm{~mm}$. O peso (P) e a massa muscular esquelética (MME) foram avaliados com a bioimpedância octopolar InBody720 (Biospace Co. Ltd, Seoul, Coreia), respeitando os procedimentos referidos no manual do equipamento (Biospace, 2004) e as normas de preparação expressas na literatura (Chumlea \& Sun, 2005). Este equipamento emprega oito elétrodos (4 posicionados sobre as palmas da mãos e dos polegares e, os outros quatro, nos 
calcanhares e na parte anterior dos pés) possibilitando a análise da impedância de 5 regiões do corpo (membros superiores, tronco e membros inferiores), através da medição da resistência a 6 frequências $(1 \mathrm{kHz}, 5 \mathrm{kHz}, 50$ $\mathrm{kHz}, 250 \mathrm{kHz}, 500 \mathrm{kHz}$ e $1000 \mathrm{kHz}$ ) e a reatância a três frequências $(5 \mathrm{kHz}, 50 \mathrm{kHz}$ e $250 \mathrm{kHz}$ ). As avaliações foram realizadas pelo mesmo técnico e os pontos de contacto do corpo com os elétrodos foram previamente limpos com um tecido eletrolítico recomendado pelo fabricante. Os dados foram eletronicamente importados para o Excel, utilizando o software Lookin'Body 3.0 (Biospace Co. Ltd, Seoul, Coreia). $\mathrm{O}$ índice de massa muscular esquelética $[\mathrm{IMME}=(\mathrm{MME} / \mathrm{P}) \times 100]$ foi calculado de acordo com a fórmula proposta por Janssen, Heymsfield, e Ross (2002), considerando-se existir sarcopenia na presença de IMME $\leq 28 \%$. A validade do InBody 720 na estimação da composição corporal total e segmentar é documentada na literatura (Ling et al., 2011). Os erros técnicos do P, da ALT e da MME, obtidos com base na realização de medições em duplicado em 10 mulheres pósmenopáusicas e através da aplicação da fórmula $\mathrm{ET}=\left(\sum \mathrm{d}^{2} / 2 \mathrm{n}\right)^{0,5}(d$, diferença entre as duas avaliações; $n$, número de elementos da amostra), foram respetivamente os seguintes: $0.06 \mathrm{~kg}, 0.09 \mathrm{~cm}$ e $0.20 \mathrm{~kg}$.

A atividade física foi avaliada através da versão longa do International Physical Activity Questionnaire (IPAQ), reunindo questões relacionadas com a frequência e a duração das atividades físicas realizadas por mais de dez minutos contínuos durante a última semana e abrangendo 4 domínios de atividade física (TRABALHO, TRANSPORTE ATIVO, ATIVIDADES DOMÉSTICAS E JARDINAGEM, LAZER). Cada um dos referidos domínios foi expresso em minutos/semana através da multiplicação da frequência semanal pela duração de cada uma das atividades realizadas. Para o cálculo do gasto energético procedeu-se à multiplicação do valor do dispêndio de energia de acordo com a atividade realizada, considerando-se a sua duração e frequência semanal (tempo em minutos/semana). A conversão dos dados obtidos pelo IPAQ em medida de equivalente metabólico (METs) foi realizada considerando os seguintes coeficientes: TRABALHO (3.3 METs, caminhada; 4.0 METs, atividade moderada; 8.0 METs, atividade vigorosa), TRANSPORTE ATIVO (3.3 METs, caminhada; 6.0 METs, deslocação com bicicleta), ATIVIDADES DOMÉSTICAS E JARDINAGEM (4.0 METs, atividade moderada no jardim/quintal; 3.0 METs, atividade moderada dentro de casa; 5.5 METs, atividade vigorosa no jardim/quintal) e TEMPO LIVRE (3.3 METs, caminhada; 4.0 METs, atividade moderada; 8.0 METs, atividade vigorosa). O produto do valor de METs da AF pela sua duração e frequência resultou no gasto calórico em METs minutos/semana e a sua conversão em $\mathrm{kcal} / \mathrm{semana}$ foi obtida através da fórmula: METs-minutos/semana $\times$ Peso $(\mathrm{kg}) / 60 . \mathrm{O}$ questionário IPAQ foi aplicado e analisado pelo mesmo técnico e o seu processamento obedeceu às diretrizes disponibilizadas no site www.ipaq.ki.se. A validade e a confiabilidade deste questionário estão documentadas na literatura (Craig et al., 2003).

\section{Análise Estatística}

Os dados foram analisados recorrendo ao programa estatístico SATA (versão 7.0. Stata Corp., College Station, USA). O poder discriminatório e os pontos de corte da atividade física para prevenção da sarcopenia foram analisados através de curvas Receiver Operating Characteristic (ROC).

Inicialmente, procedeu-se à identificação da área total sob a curva ROC entre a $\mathrm{AF}$ e a prevenção da sarcopenia estando uma maior área associada a um poder discriminatório mais destacado. Nesta etapa utilizou-se intervalo de confiança (IC) a 95\%. O cálculo do IC a $95 \%$ determina se a capacidade preditiva da $\mathrm{AF}$ não é ao acaso, não devendo o seu limite inferior ser menor do que 0.50 . Na sequência, foram calculadas a sensibilidade e a especificidade, além dos pontos de corte da atividade física para a prevenção da sarcopenia. Procedeu-se posteriormente à realização da estratificação para verificação da modificação do efeito e confundimemto sendo observadas as medidas pontuais estrato-específicas e os seus intervalos de confiança. Considerando-se um intervalo de 
confiança de $95 \%$ pelo método de MantelHaenzel, a análise para confundimento foi executada comparando-se a odds ratio (OR) entre a associação bruta e a ajustada pelos possíveis confundidores, sendo considerado o valor de $10 \%$ como parâmetro de identificação da diferença entre as referidas associações.

A análise por meio de regressão logística foi realizada recorrendo ao método backword, partindo-se do modelo completo e retirando-se uma a uma as possíveis variáveis de confundimento, que, quando suprimidas do modelo, poderiam causar alteração igual ou superior a $10 \%$ na medida pontual de associação entre a atividade física e a sarcopenia (Hosmer \& Lemeshow, 2005). Por último, foi estimada a OR por meio do modelo que melhor explicava a associação. As covariáveis analisadas como possíveis modificadoras de efeito e confundidoras foram as seguintes: idade, tempo de menopausa, terapia hormonal, natureza da menopausa $(0$, natural; 1, induzida), regularidade dos ciclos menstruais e utilização de métodos contracetivos.

A atividade física foi categorizada de acordo com os pontos de corte identificados neste estudo para o dispêndio calórico semanal na prática de caminhada $(0,<580 \mathrm{kcal} / \mathrm{semana} ; 1$, $\geq 580 \mathrm{kcal} / \mathrm{semana} ; 0,<816 \mathrm{kcal} / \mathrm{semana} ; 1$, $\geq 816 \mathrm{kcal} / \mathrm{semana}$ ) não tendo sido identificados pontos de corte para a atividade física moderada e vigorosa.

\section{RESULTADOS}

A idade média da amostra foi de 57.2 (6.6) anos revelando a maioria dos seus elementos uma menopausa natural (75.2\%), um tempo de menopausa igual ou inferior a 10 anos (59.2\%) e o uso de terapia hormonal (Tabela 1). A utilização de métodos contracetivos e a regularidade de períodos menstruais foram documentadas, respetivamente, por $71.6 \%$ e $85 \%$ das mulheres. O valor de tendência central do gasto energético semanal foi de 5411.5 $\mathrm{kcal} / \mathrm{semana}$, sendo obtido, sobretudo, com a prática de atividade física de intensidade moderada (3134.8 kcal/semana). A MME apresentou uma média de 22.3 (2.8) kg, com apenas $9.3 \%$ das mulheres a serem classificadas como sarcopénicas. A Tabela 2 ilustra as áreas sob a curva ROC entre a atividade física e a ausência de sarcopenia.

Tabela 1

Análise descritiva da amostra $(n=257)$.

\begin{tabular}{lcc}
\hline \multicolumn{1}{c}{ Variáveis } & $\begin{array}{c}\text { Média/Mediana* DP ou } \\
\text { percentagem }\end{array}$ & $\begin{array}{c}\text { Amplitude ou intervalo de } \\
\text { confiança }\end{array}$ \\
\hline Idade (anos) & $57.2 \pm 6.6$ & $40.6-79.6$ \\
Peso (kg) & $69.1 \pm 11.3$ & $45.8-108.7$ \\
Altura (cm) & $155.0 \pm 5.2$ & $142-170$ \\
Massa Muscular Esquelética (kg) & $22.3 \pm 2.8$ & $15.8-30.4$ \\
Índice de Massa Muscular Esquelética (\%) & & \\
Atividade Física Habitual (kcal/semana) & $1724.4^{*} \pm 2549.9$ & $0-15178.1$ \\
$\quad$ Caminhada & $3134.8^{*} \pm 4213.8$ & $0-25765.5$ \\
Moderada & $552.2^{*} \pm 2467.1$ & $0-22304.8$ \\
Vigorosa & $5411.5^{*} \pm 6937.2$ & $0-45591.1$ \\
Total & & $(10.9-20.3)$ \\
Regularidade dos Períodos Menstruais (\%) & 15.0 & $(80.2-89.4)$ \\
Não & 85.0 & $(22.9-34.4)$ \\
Sim & & $(65.6-77.1)$ \\
Uso de Métodos Contracetivos (\%) & 28.4 & $(39.7-52.4)$ \\
Não & 71.6 & $(47.6-60.3)$ \\
Sim & & \\
Terapia Hormonal (\%) & 45.9 & $(52.8-65.3)$ \\
Não & 54.1 & $(34.6-47.2)$ \\
Sim & & $(69.3-80.4)$ \\
Tempo de menopausa (\%) & & $(19.6-30.6)$ \\
I0 anos & 59.2 &
\end{tabular}


Tabela 2

Áreas sob a curva Receiver Operating Characteristics e intervalo de confiança a $95 \%$ da quantidade de atividade física (kcal/semana) relacionada com a prevenção da sarcopenia.

\begin{tabular}{lc}
\hline \multicolumn{1}{c}{ Atividade Física } & AUC \\
\hline Caminhada & $0.62(0.51-0.74)^{*}$ \\
Moderada & $0.58(0.46-0.71)$ \\
Vigorosa & $0.49(0.42-0.56)$ \\
Total & $0.59(0.47-0.71)$ \\
\hline
\end{tabular}

* Área sob a curva ROC apresentando poder discriminatório para a ausência de sarcopenia (Li-IC $\geq 0.50$ )

Apenas a atividade física de caminhada se revelou um discriminador significativo ( $\mathrm{p} \leq$ 0.05) da sarcopenia sendo a área sob as curvas ROC de 0.62 (0.51 - 0.74). Os níveis de atividade física de intensidade moderada, vigorosa e total, não evidenciaram poder discriminatório para a ausência da sarcopenia, sendo os respetivos intervalos de confiança das curvas ROC inferior a 0.50. A atividade física de caminhada revelou-se suficiente para proteção da sarcopenia em mulheres pós-menopausicas.

A Tabela 3 expõe os pontos de corte definidos no estudo para atividade física de caminhada como discriminadores da sarcopenia com as suas respetivas sensibilidades e especificidades. A caminhada, com um correspondente gasto energético de 580 $\mathrm{kcal} / \mathrm{semana}$ a $816 \mathrm{kcal} / \mathrm{semana}$, exibiu melhores resultados para a prevenção da sarcopenia. A análise da associação dos níveis da caminhada revelou que apenas o gasto energético de $816 \mathrm{kcal} / \mathrm{semana}$ mostrou associação para proteção da sarcopenia, independente da idade, das características da menopausa e dos aspetos relacionados com o período reprodutivo da mulher.

Tabela 3

Sensibilidade e especificidade da quantidade de atividade física (AF, kcal/semana) requerida para a prevenção da sarcopenia e associação entre a AF e a sarcopenia.

\begin{tabular}{lcccc}
\hline Atividade Física & Ponto de corte & Sensibilidade & Especificidade & OR (IC 95\%) \\
\hline Caminhada & 580 & 56 & 71 & 0.51 \# $(0.20-1.31)$ \\
Moderada & 816 & 51 & 75 & $0.29 *(0.10-0.83)$ \\
Vigorosa & $\mathrm{NR}$ & ----- & --- & \\
Total & $\mathrm{NR}$ & ---- & --- & \\
NR - indicador não recomendável para a prevenção da sarcopenia (pontos de corte não apresentam boa \\
sensibilidade e especificidade). \# Associação bruta; * Associação ajustada por tempo de menopausa e \\
regularidade dos ciclos menstruais
\end{tabular}

\section{DISCUSSÃO}

O presente estudo foi realizado visando contribuir para a definição de níveis de atividade física adequados para a prevenção da sarcopenia em mulheres pós-menopáusicas. A pesquisa revelou que a prática de caminhada com um gasto calórico semanal entre 580 a $816 \mathrm{kcal}$, previne a sarcopenia em mulheres nesta fase do climatério. Os dados evidenciaram também que a associação do gasto energético de $816 \mathrm{kcal} / \mathrm{semana}$ com a sarcopenia é independente da idade, das caraterísticas da menopausa (tempo, natureza e terapia hormonal) e de alguns aspetos acomunados ao período reprodutivo da mulher (uso de métodos contracetivos e regularidade dos ciclos menstruais).

As pesquisas apontam que as mulheres pósmenopáusicas ativas exibem maior proteção para doenças metabólicas e cardiovasculares e alterações mais favoráveis na composição corporal (Silva, Alves, Maturana, \& Spritzer, 2013). É também observada uma associação inversa da atividade física com a obesidade e alguns indicadores antropométricos da adiposidade central como o perímetro da cintura e o índice cintura-anca (Colpani, Oppermann, \& Spritzer, 2013). Independentemente dos níveis de adiposidade total e intra-abdominal, a atividade de caminhada e moderada está relacionada a uma menor manifestação da síndrome metabólica (Bao et al., 2013) e de doenças cardiovasculares na pós-menopausa (Perry et al., 2013).

A maior frequência de atividade física no tempo de lazer durante a fase adulta está associada a uma menor massa gorda total e a 
níveis acrescidos de massa muscular apendicular na mulher, reforçando a necessidade de atividades motoras em níveis de intensidade leve e moderada-vigorosa (Bann et al., 2014). As alterações da adiposidade na mulher ocorrem particularmente no período que inclui a transição da menopausa e o primeiro ano após a instalação da amenorreia permanente (Tchernof \& Poehlman, 1998) gerando o aumento de citocinas pro-inflamatórias como a interleucina 6 e o fator de necrose tumoral alfa, ambas produzidas nas células adiposas, expandindo o catabolismo das proteínas (Rolland \& Vellas, 2009). Este facto justifica a pertinência da implementação de programas de controlo do peso no período imediatamente antes do final do período menstrual e nos estádios iniciais da pósmenopausa.

De acordo com Riesco et al. (2010) a caminhada realizada a uma intensidade de $60 \%$ da frequência cardíaca de reserva promove um aumento significativo na aptidão cardiorrespiratória de mulheres pósmenopáusicas. Na nossa investigação, a caminhada com um correspondente dispêndio energético de $816 \mathrm{kcal}$ revelou uma associação significativa com a sarcopenia, constituindo uma proposta atraente e acessível para a promoção de um estilo de vida mais ativo nesta etapa do climatério.

$\mathrm{O}$ treino de resistência muscular $\mathrm{e}$ as atividades aeróbias além dos 60 anos estão associados a uma melhoria da qualidade muscular na mulher (Barbat-Artigas, Dupontgand, Pion, Feiter-Murphy, \& AubertinLeheudre, 2014). De acordo com Kemmler e von Stengel (2013), a sua combinação proporciona melhores resultados na massa muscular esquelética apendicular e na massa magra em mulheres com mais de 60 anos. Park, Park, Shephard, e Aoyagi (2010) demonstraram que a realização de 7000 a 8000 passos diários e a acumulação de 15 a 20 minutos por dia de atividade física de intensidade moderada estavam associados a uma melhor condição muscular nas mulheres pós-menopáusicas.

Garber et al. (2011) sugerem que o exercício cardiovascular de intensidade moderada-vigorosa deve permitir obter um dispêndio energético igual ou superior a 500-1000 $\mathrm{MET} / \mathrm{minutos} /$ semana e que o treino de resistência muscular deve envolver grandes grupos musculares e exercícios de equilíbrio, agilidade e coordenação (prevenção do risco de queda). Chiang, Wahlqvist, Huang, e Chang, (2013) destacam a necessidade de obtenção de um dispêndio energético semanal entre 300 a 2000 kcal para a preservação da condição muscular na mulher pós-menopáusica.

Uma possível limitação do estudo pode ser atribuída ao instrumento usado para avaliar a duração e intensidade da atividade física (IPAQ) que apesar de ser utilizado em diversos estudos nacionais e internacionais pode provocar erros de classificação, considerando que os instrumentos do tipo questionário aplicados na forma de entrevista podem provocar viés de memória. Além disto, os coeficientes dos METs de referência do dispêndio energético em geral são subestimados quando utilizados em populações com idade semelhante ao presente estudo pelo fato do compêndio de atividade física ter sido desenvolvido com base em população de adultos jovens.

\section{CONCLUSÃo}

Os resultados do presente estudo sugerem que a prática da atividade física na forma de caminhada reunindo um gasto calórico de aproximadamente $800 \mathrm{kcal} / \mathrm{semana}$, previne a sarcopenia em mulheres pós-menopáusicas.

\section{Agradecimentos:}

Nada a declarar.

\section{Conflito de Interesses:}

Nada a declarar.

\footnotetext{
Financiamento:

Investigação suportada pela Fundação para a Ciência e Tecnologia (POCI/DES/59049/2004) e pelo Programa Operacional para a Ciência e a Inovação 2010 (POCI2010), co-financiado pelo Fundo Social Europeu (FEDER).
} 


\section{REFERÊNCIAS}

Aloia, J. F., McGowan, D. M., Vaswani, A. N., Ross, P., \& Cohn, S. H. (1991). Relationship of menopause to skeletal and muscle mass. The American Journal of Clinical Nutrition, 53(6), 1378-1383.

Bann, D., Kuh, D., Wills, A. K., Adams, J., Brage, S., \& Cooper, R. (2014). Physical activity across adulthood in relation to fat and lean body mass in early old age: findings from the Medical Research Council National Survey of Health and Development, 1946-2010. American Journal of Epidemiology, 179(10), 1197-1207. http://doi.org/10.1093/aje/kwu033

Bao, P.-P., Zheng, Y., Nechuta, S., Gu, K., Cai, H., Peng, P., ... Lu, W. (2013). Exercise after diagnosis and metabolic syndrome among breast cancer survivors: a report from the Shanghai Breast Cancer Survival Study. Cancer Causes \& Control: $\quad$ CCC, 24(9), 1747-1756. http://doi.org/10.1007/s10552-013-0252-7

Barbat-Artigas, S., Dupontgand, S., Pion, C. H., FeiterMurphy, Y., \& Aubertin-Leheudre, M. (2014). Identifying recreational physical activities associated with muscle quality in men and women aged 50 years and over. Journal of Cachexia, Sarcopenia and Muscle, 5(3), 221-228. http://doi.org/10.1007/s13539-014-0143-0

Barsalani, R., Brochu, M., \& Dionne, I. J. (2013). Is there a skeletal muscle mass threshold associated with the deterioration of insulin sensitivity in sedentary lean to obese postmenopausal women? Diabetes Research and Clinical Practice, 102(2), 123-128.

http://doi.org/10.1016/j.diabres.2013.09.008

Biospace. (2004). Inbody 720, the precision body composition analyser: user's guide. Seoul: Biospace Co., Ltd.

Boirie, Y. (2009). Physiopathological mechanism of sarcopenia. The Journal of Nutrition, Health \& Aging, 13(8), 717-723.

Canário, A. C. G., Cabral, P. U., Spyrides, M. H., Giraldo, P. C., Eleutério, J., \& Gonçalves, A. K. (2012). The impact of physical activity on menopausal symptoms in middle-aged women. International Journal of Gynaecology and Obstetrics: The Official Organ of the International Federation of Gynaecology and Obstetrics, 118(1), 34-36. http://doi.org/10.1016/j.ijgo.2012.02.016

Chiang, P.-H., Wahlqvist, M. L., Huang, L.-Y., \& Chang, Y.-C. (2013). Leisure time physical activities and dietary quality of the general and indigenous Taiwanese populations are associated with fat distribution and sarcopenia. Asia Pacific Journal of Clinical Nutrition, 22(4), 599-613. http://doi.org/10.6133/apjcn.2013.22.4.19

Chumlea, W. C., \& Sun, S. (2005). Bioelectrical impedance analysis. Em S. Heymsfield, T. G. Lohman, Z. Wang, \& S. B. Going (Eds.), Human Body Composition (pp. 79-88). Champagn, IL: Human Kinetics.
Chung, J.-Y., Kang, H.-T., Lee, D.-C., Lee, H.-R., \& Lee, Y.-J. (2013). Body composition and its association with cardiometabolic risk factors in the elderly: a focus on sarcopenic obesity. Archives of Gerontology and Geriatrics, 56(1), 270-278. http://doi.org/10.1016/j.archger.2012.09.007

Clark, B. C., \& Taylor, J. L. (2011). Age-related changes in motor cortical properties and voluntary activation of skeletal muscle. Current Aging Science, 4(3), 192-199. http://doi.org/10.2174/1874609811104030192

Colpani, V., Oppermann, K., \& Spritzer, P. M. (2013). Association between habitual physical activity and lower cardiovascular risk in premenopausal, perimenopausal, and postmenopausal women: a population-based study. Menopause (New York, N.Y.), 20(5), 525-531. http://doi.org/10.1097/GME.0b013e318271b38 8

Craig, C. L., Marshall, A. L., Sjöström, M., Bauman, A. E., Booth, M. L., Ainsworth, B. E., ... Oja, P. (2003). International physical activity questionnaire: 12-country reliability and validity. Medicine and Science in Sports and Exercise, 35(8),

1381-1395. http://doi.org/10.1249/01.MSS.0000078924.61 453.FB

Cruz-Jentoft, A. J., Baeyens, J. P., Bauer, J. M., Boirie, Y., Cederholm, T., Landi, F., ... Zamboni, M. (2010). Sarcopenia: European consensus on definition and diagnosis: Report of the European Working Group on Sarcopenia in Older People. Age and Ageing, 39(4), 412-423. http://doi.org/10.1093/ageing/afq034

Garber, C. E., Blissmer, B., Deschenes, M. R., Franklin, B. A., Lamonte, M. J., Lee, I.-M., ... Swain, D. P. (2011). American College of Sports Medicine position stand. Quantity and quality of exercise for developing and maintaining cardiorespiratory, musculoskeletal, and neuromotor fitness in apparently healthy adults: guidance for prescribing exercise. Medicine and Science in Sports and Exercise, 43(7), 13341359.

http://doi.org/10.1249/MSS.0b013e318213fefb

Gonçalves, A. K. da S., Canário, A. C. G., Cabral, P. U., da Silva, R. A. H., Spyrides, M. H. C., Giraldo, P. C., \& Eleutério, J. (2011). [Impact of physical activity on quality of life in middle-aged women: a population based study]. Revista Brasileira De Ginecologia E Obstetrícia: Revista Da Federação Brasileira Das Sociedades De Ginecologia $E$ Obstetrícia, 33(12), 408-413.

Heyward, V. H., \& Wagner, D. R. (2004). Applied Body Composition Assessment (2nd ed.). Champagn, IL: Human Kinetics.

Hosmer, D. W., \& Lemeshow, S. (2005). Applied Logistic Regression (2nd ed.). New York: John Wiley \& Sons.

Janssen, I., Heymsfield, S. B., \& Ross, R. (2002). Low relative skeletal muscle mass (sarcopenia) in 
older persons is associated with functional impairment and physical disability. Journal of the American Geriatrics Society, 50(5), 889-896. http://doi.org/10.1046/j.1532-

5415.2002.50216.x

Kemmler, W., \& von Stengel, S. (2013). Exercise frequency, health risk factors, and diseases of the elderly. Archives of Physical Medicine and Rehabilitation, 94(11), 2046-2053. http://doi.org/10.1016/j.apmr.2013.05.013

Lee, J.-Y., \& Lee, D.-C. (2013). Muscle strength and quality are associated with severity of menopausal symptoms in peri- and postmenopausal women. Maturitas, 76(1), 88-94. http://doi.org/10.1016/j.maturitas.2013.06.007

Ling, C. H. Y., de Craen, A. J. M., Slagboom, P. E., Gunn, D. A., Stokkel, M. P. M., Westendorp, R. G. J., \& Maier, A. B. (2011). Accuracy of direct segmental multi-frequency bioimpedance analysis in the assessment of total body and segmental body composition in middle-aged adult population. Clinical Nutrition (Edinburgh, Scotland), 30(5), 610-615. http://doi.org/10.1016/j.clnu.2011.04.001

Maltais, M. L., Desroches, J., \& Dionne, I. J. (2009). Changes in muscle mass and strength after menopause. Journal of Musculoskeletal \& Neuronal Interactions, 9(4), 186-197.

Monteiro, M., Gabriel, R., Aranha, J., Neves e Castro, M., Sousa, M., \& Moreira, M. (2010). Influence of obesity and sarcopenic obesity on plantar pressure of postmenopausal women. Clinical Biomechanics, 25(5), 461-467. http://doi.org/10.1016/j.clinbiomech.2010.01.0 17

Moreira, H., Castro, R., Freitas, J., Gabriel, R., Monteiro, M., \& Machado, M. (2008). Functional fitness, obesity and sarcopenia in postmenopausal women. Climacteric, 11(Supp $1)$, S120. http://doi.org/10.1080/13697130802080966

Nedergaard, A., Henriksen, K., Karsdal, M. A., \& Christiansen, C. (2013). Musculoskeletal ageing and primary prevention. Best Practice \& Research. Clinical Obstetrics \& Gynaecology, 27(5), 673-688. http://doi.org/10.1016/j.bpobgyn.2013.06.001

Park, H., Park, S., Shephard, R. J., \& Aoyagi, Y. (2010). Yearlong physical activity and sarcopenia in older adults: the Nakanojo Study. European Journal of Applied Physiology, 109(5), 953-961. http://doi.org/10.1007/s00421-010-1424-8

Perry, C. K., Herting, J. R., Berke, E. M., Nguyen, H. Q., Vernez Moudon, A., Beresford, S. A. A., ... Lacroix, A. Z. (2013). Does neighborhood walkability moderate the effects of intrapersonal characteristics on amount of walking in postmenopausal women? Health \& Place, 21, 39-45. http://doi.org/10.1016/j.healthplace.2012.12.00 7

Riesco, E., Tessier, S., Pérusse, F., Turgeon, S., Tremblay, A., Weisnagel, J., ... Mauriège, P. (2010). Impact of walking on eating behaviors and quality of life of premenopausal and early postmenopausal obese women. Menopause (New York, N.Y.), 17(3), 529-538. http://doi.org/10.1097/gme.0b013e3181d1236 1

Rolland, Y., \& Vellas, B. (2009). [Sarcopenia]. La Revue De Médecine Interne, 30(2), 150-160. http://doi.org/10.1016/j.revmed.2008.08.013

Silva, T. R., Alves, B. C., Maturana, M. A., \& Spritzer, P. M. (2013). Healthier dietary pattern and lower risk of metabolic syndrome in physically active postmenopausal women. Journal of the American College of Nutrition, 32(5), 287-295. http://doi.org/10.1080/07315724.2013.826111

Tan, M. N., Kartal, M., \& Guldal, D. (2014). The effect of physical activity and body mass index on menopausal symptoms in Turkish women: a cross-sectional study in primary care. $B M C$ Women's Health, 14(1), 38. http://doi.org/10.1186/1472-6874-14-38

Tchernof, A., \& Poehlman, E. T. (1998). Effects of the menopause transition on body fatness and body fat distribution. Obesity Research, 6(3), 246254.

Vagetti, G. C., Barbosa Filho, V. C., Moreira, N. B., de Oliveira, V., Mazzardo, O., \& de Campos, W. (2015). The Association Between Physical Activity and Quality of Life Domains Among Older Women. Journal of Aging and Physical Activity, 23(4), 524-533. http://doi.org/10.1123/japa.2013-0070

Vaidya, R. (2014). Obesity, sarcopenia and postmenopausal osteoporosis: An interlinked triad! Journal of Mid-Life Health, 5(1), 1-2. http://doi.org/10.4103/0976-7800.127778

Vilaça, K. H. C., Carneiro, J. A. O., Ferriolli, E., Lima, N. K. da C., de Paula, F. J. A., \& Moriguti, J. C. (2014). Body composition, physical performance and muscle quality of active elderly women. Archives of Gerontology and Geriatrics, 59(1), 44-48.

http://doi.org/10.1016/j.archger.2014.02.004

Wee, J., Sng, B. Y. J., Shen, L., Lim, C. T., Singh, G., \& Das De, S. (2013). The relationship between body mass index and physical activity levels in relation to bone mineral density in premenopausal and postmenopausal women. Archives of Osteoporosis, 8, 162. http://doi.org/10.1007/s11657-013-0162-z

World Medical Association. (2013). WMA Declaration of Helsinki: ethical principles for medical research involving human subjects (64th WMA General Assembly). Fortaleza, CE: World Medical Association. 